\title{
The effect of intervention by an antimicrobial stewardship team on anaerobic bacteremia
}

Gaku Kuwabara, Satomi Yukawa, Koichi Yamada, Waki Imoto, Kazushi Yamairi, Wataru Shibata, Naoko Yoshii, Tetsuya Watanabe, Kazuhisa Asai, Makoto Niki, Kiyotaka Nakai, Yasuyo Okada, Akiko Fujita, Yukihiro Kaneko, Tomoya Kawaguchi, Hiroshi Kakeya

\begin{tabular}{|c|l|}
\hline Citation & Anaerobe. $64 ; 102214$ \\
\hline Issue Date & $2020-08$ \\
\hline Type & Journal Article \\
\hline Textversion & author \\
\hline Highlights & $\begin{array}{l}\text {-Antimicrobial stewardship decreased carbapenem use in anaerobic bacteremia } \\
\text {-Antimicrobial stewardship interventions did not worsen patient outcomes } \\
\text { bacteremia } \\
\text { Empiric tazobactam/piperacillin use increased after antimicrobial stewardship }\end{array}$ \\
\hline \multirow{2}{*}{ Rights } & $\begin{array}{l}\text { C2020 Elsevier. This manuscript version is made available under the } \\
\text { CC-BY-NC-ND } 4.0 \text { License. http://creativecommons.org/licenses/by-nc-nd/4.0/ } \\
\text { This is the accepted manuscript version. For Academic research only. } \\
\text { The article has been published in final form at } \\
\text { https://doi.org/10.1016/j.anaerobe.2020.102214. } \\
\text { Please cite only the published version. }\end{array}$ \\
\hline DOI & \begin{tabular}{l} 
10.1016/j.anaerobe.2020.102214 \\
\hline
\end{tabular} \\
\hline
\end{tabular}

\section{Self-Archiving by Author(s)}

Placed on: Osaka City University Repository 


\section{The effect of intervention by an antimicrobial stewardship team on anaerobic}

\section{bacteremia}

Gaku Kuwabara $^{\text {a,b,c }}$, Satomi Yukawaa,b,c, Koichi Yamada ${ }^{a, b, d}$, Waki Imoto ${ }^{\text {a,b,c }}$, Kazushi Yamairi $^{\mathrm{a}, \mathrm{b}}$, Wataru Shibata ${ }^{\mathrm{a}, \mathrm{b}}$, Naoko Yoshii ${ }^{\mathrm{a}, \mathrm{b}}$, Tetsuya Watanabe ${ }^{\mathrm{c}}$, Kazuhisa Asai ${ }^{\mathrm{c}}$, Makoto Niki ${ }^{\text {, }}$ Kiyotaka Nakaie ${ }^{\mathrm{b}}$, Yasuyo Okada ${ }^{\mathrm{b}}$, Akiko Fujita $^{\mathrm{b}}$, Yukihiro Kaneko ${ }^{\mathrm{d}, \mathrm{e}}$, Tomoya Kawaguchi ${ }^{\mathrm{c}}$, and Hiroshi Kakeya ${ }^{\mathrm{a}, \mathrm{b}, \mathrm{d}}$

a Department of Infection Control Science, Graduate School of Medicine, Osaka City

University, 1-4-3, Asahi-machi, Abeno-ku, Osaka-shi, Osaka, 545-8585 Japan

${ }^{b}$ Department of Infection Control and Prevention, Osaka City University Hospital, 1-4-3,

Asahi-machi, Abeno-ku, Osaka-shi, Osaka, 545-8585 Japan

'Department of Respiratory Medicine, Graduate School of Medicine, Osaka City

University, 1-4-3, Asahi-machi, Abeno-ku, Osaka-shi, Osaka, 545-8585 Japan

${ }^{\mathrm{d}}$ Research Center for Infectious Diseases, Graduate School of Medicine, Osaka City

University, 1-4-3, Asahi-machi, Abeno-ku, Osaka-shi, Osaka, 545-8585 Japan

${ }^{\mathrm{e} B a c t e r i o l o g y, ~ O s a k a ~ C i t y ~ U n i v e r s i t y ~ H o s p i t a l, ~ 1-4-3, ~ A s a h i-m a c h i, ~ A b e n o-k u, ~}$

Osaka-shi, Osaka, 545-8585 Japan 
Corresponding author: Hiroshi Kakeya

Department of Infection Control Science, Graduate School of Medicine, Osaka City

University, 1-4-3, Asahi-machi, Abeno-ku, Osaka-shi, Osaka, 545-8585 Japan

Tel: $+81-66-645-3784$

Fax: +81-66-646-6056

E-mail: kakeya@med.osaka-cu.ac.jp 


\section{Abstract}

The effect of antimicrobial stewardship (AS) on anaerobic bacteremia is uncertain. This study aimed to assess the effect of interventions by the AS team (AST) on clinical and microbiological outcomes and antimicrobial use. An AS program was introduced at Osaka City University Hospital in January 2014; an interdisciplinary AST was established. We enrolled patients with anaerobic bacteremia between January 2009 and December 2018. Patients were classified into the pre-intervention group (from January 2009 to December 2013) and the post-intervention group (from January 2014 to December 2018). A significant decrease in definitive carbapenem use $(\mathrm{P}=0.0242)$ and an increase in empiric tazobactam/piperacillin use $(\mathrm{P}=0.0262)$ were observed in the post-intervention group. The de-escalation rate increased significantly from $9.38 \%$ to $32.7 \%(\mathrm{P}=0.0316)$ in the post-intervention group. The susceptibility of Bacteroides species and 30-day mortality did not worsen in the post-intervention group. These results showed that interventions by an AST can reduce carbapenem use and increase the de-escalation rate without worsening patient outcomes.

Keywords: antimicrobial stewardship, anaerobic bacteremia, Bacteroides species, carbapenem, de-escalation 


\section{Abbreviations}

AS, antimicrobial stewardship; ASP, antimicrobial stewardship program; AST, antimicrobial stewardship team; CRE, carbapenem-resistant Enterobacteriaceae; TAZ/PIPC, tazobactam/piperacillin; WBC, white blood cell 


\section{Introduction}

Antimicrobial consumption has been increasing globally [1]. Although antimicrobials are essential for medical care, misuse and overuse are risk factors for the emergence of antimicrobial resistance. Moreover, $20-50 \%$ of all antimicrobials prescribed in acute care hospitals are reported to be either unnecessary or inappropriate [2]. Infections caused by resistant bacteria lead to poor outcomes, increased costs and have become a serious threat to public health worldwide [3, 4]. Furthermore, in 2013, the Centers for Disease Control and Prevention reported that approximately 2 million patients in the United States are infected with resistant bacteria annually and at least 23,000 of those patients die [5]. Considering the slow speed of development of new antimicrobials and the immediate emergence of resistant bacteria after the development of new antimicrobials [6], it is vital to make the best use of currently available antimicrobials and prevent the emergence of resistant bacteria. To manage this problem, antimicrobial stewardship (AS) was proposed by the Infectious Diseases Society of America and the Society for Healthcare Epidemiology of America [7].

Anaerobic bacteremia is uncommon, accounting for only $0.5-13 \%$ of all positive blood cultures [8]. However, anaerobic bacteremia can cause multiorgan failure [9] and is associated with significantly poor outcomes with a mortality rate of $13-45 \%$ 
[8]. Furthermore, the incidence of anaerobic bacteremia has been increasing gradually [10] presumably reflecting an increase in the number of patients with complex medical history such as chemotherapy, organ transplantation and immunosuppression due to various drugs or diseases [11]. Moreover, antimicrobial resistance among anaerobic bacteria has been increasing [12-16]. Thus, anaerobic bacteremia should be recognized as an important infection.

Although previous reports showed that AS improves patient outcomes by reducing inappropriate antimicrobial use, antimicrobial consumption, costs, resistant bacteria and length of hospital stay $[2,17,18]$, the effect of an AS program (ASP) on anaerobic infections is uncertain.

An ASP was introduced at Osaka City University Hospital in January 2014, and we established an interdisciplinary AS team (AST) [19]. The purpose of this study was to assess the effect of interventions by the AST on the outcomes of anaerobic bacteremia.

\section{Materials and methods}

2.1 Study design and setting

This retrospective observational study was conducted at Osaka City University 
Hospital, a 980-bed tertiary-care hospital in Osaka, Japan, and included patients with positive blood cultures for anaerobic bacteria admitted to the hospital between January 2009 and December 2018. Patients admitted between January 2009 and December 2013 were classified into the pre-intervention group, and those admitted between January 2014 and December 2018 were classified into the post-intervention group. All data were collected from electronic medical records. The Ethics Committee of Osaka City University Graduate School of Medicine approved this study (number: 2019-048), and it was performed in accordance with the principles of the Declaration of Helsinki. The need for written informed consent was waived owing to the retrospective nature of the study.

\subsection{Intervention}

An ASP was introduced at our institution in January 2014, and its core strategy was prospective audit and feedback [19]. The activities of the AST included 1) sharing of information about new positive blood cultures and the detection of resistant bacteria from other sites by microbiological technologists at meetings each morning, 2) regarding patients with positive blood culture and patients administered inappropriate or broad-spectrum antimicrobials, recommendations by an infectious disease physician for 
alteration or de-escalation of antimicrobials and additional cultures, 3) therapeutic drug monitoring of anti-methicillin-resistant Staphylococcus aureus drugs and compiling a list of long-term use of broad-spectrum antimicrobials (more than 7 days) by pharmacists, 4) hospital rounds focused on patients with positive blood cultures or long-term antimicrobial prescriptions, and 5) a meeting every Tuesday to determine treatment policies.

\subsection{Definition of anaerobic bacteremia}

Patients with positive blood cultures for anaerobic bacteria were identified retrospectively. Clinically significant anaerobic bacteremia was defined as $\geq 1$ positive blood cultures with $\geq 1$ of the following criteria: white blood cell (WBC) count $<4,000$ or $>12,000 / \mu \mathrm{L}$; body temperature $>38^{\circ} \mathrm{C}$; or physical, pathological, or surgical evidence consistent with infection [20]. Considering its low pathogenicity and the infrequency of causing infections, Propionibacterium species were considered significant only when they were isolated in at least two sets of bottles drawn at the same time [21]. Cases with the same bacteria isolated multiple times within 14 days were defined as the same episode [22]. 


\subsection{Variables}

The following data were obtained for the analysis of the prognostic factors for 30-day mortality: age, sex, WBC count, C-reactive protein, Sequential Organ Failure Assessment (SOFA) scores, past abdominal surgery history, malignancy, diabetes mellitus, antineoplastic chemotherapy (within 30 days before bacteremia onset), chronic kidney disease, use of immunosuppressants, intensive care unit admission (within 24 hours after bacteremia onset), nosocomial infection, multibacterial bacteremia, inappropriate empiric therapy, and inappropriate definitive therapy. Antimicrobial treatment administered within 5 days after bacteremia onset was defined as empiric therapy, and that administered afterward was defined as definitive therapy [23]. Inappropriate therapy was defined as the failure to administer anti-anaerobic drug therapy. Anti-anaerobic antimicrobials included beta-lactam/beta-lactamase inhibitor combinations, oxacephem, carbapenem, clindamycin, and metronidazole [20]. In view of the declining activity of clindamycin against Bacteroides species, it was regarded as inappropriate therapy for this organism. Vancomycin and teicoplanin were defined as appropriate therapies for Clostridium species. Sources of bacteremia were speculated based on clinical courses, symptoms, laboratory findings, imaging findings, and cultures from other sites. De-escalation was defined as a change in the appropriate antimicrobial 
therapy from an empiric broad-spectrum therapy to a narrower spectrum therapy based on the culture results and susceptibility by changing antimicrobials and/or discontinuing combination therapy [24]. Broad-spectrum antimicrobials included carbapenems, tazobactam/piperacillin (TAZ/PIPC), fourth-generation cephalosporins, and fluoroquinolones.

2.5. Microbiological tests

The BACT/ALERT 3D Microbial System and BACT/ALERT FA Plus, FN Plus, and PF bottle (bioMérieux, Tokyo, Japan) were used for blood cultures. All blood samples were incubated for 1 week. Subculture and identification of anaerobic bacteria were performed using Brucella HK Agar (Kyokuto Pharmaceutical Industrial, Tokyo, Japan) and the BBL CRYSTAL ANR (Becton Dickinson, Tokyo, Japan), respectively. Susceptibility tests were performed only for Bacteroides species via the MicroScan WalkAway-96 SI system (Beckman Coulter, Inc., Brea, CA, USA). Susceptibility was evaluated based on the breakpoints of the Clinical and Laboratory Standards Institute 2018. 


\subsection{Statistical analyses}

Statistical analyses were performed using EZR (Saitama Medical Center, Jichi Medical University, Saitama, Japan) software. The chi-square test and Fisher's exact test were used for univariate analysis of categorical data, and the Student's t-test was used for the analysis of continuous variables. Multivariate analysis was used for logistic regression models. P-values $<0.05$ were considered statistically significant.

\section{Results}

A total of 120 patients with anaerobic bacteremia were included in the study.

The pre-intervention and post-intervention groups comprised 53 and 67 patients, respectively. Patients with multibacterial bacteremia caused by two different anaerobic bacteria or an anaerobic and an aerobic bacterium were also included. Table 1 shows the results of the univariate analysis of the characteristics of patients, including the sources of bacteremia. The characteristics of the patients were not significantly different between the two groups. Regarding the source of bacteremia, the intra-abdominal cavity was the major origin of infection in both groups. The hepatobiliary tract was also a common origin. Bacteroides species were detected most frequently, followed by Clostridium species, Fusobacterium species, Peptostreptococcus species, Prevotella 
species, Veillonella species, Propionibacterium species, and Porphyromonas species (Table2). Bacteroides species were detected significantly more frequently in the pre-intervention group. The detected rates of other bacteria were not significantly different between the two groups. The definitive use of carbapenem was significantly lower in the post-intervention group. Meanwhile, the empiric use of TAZ/PIPC was significantly higher in the post-intervention group, and the definitive use of TAZ/PIPC showed a tendency to be higher. The proportion of other antimicrobials did not change significantly. The de-escalation rate increased significantly from $9.38 \%$ to $32.7 \%$ $(\mathrm{P}=0.0316$, Table 3$)$. The duration of carbapenem and TAZ/PIPC therapy did not change (Table 4). Figure 1 describes the susceptibility rates of each drug regarding Bacteroides species, Bacteroides fragilis, and the non-fragilis group. There were no significant differences in the susceptibility of each drug between the two groups. Table 5 shows the results of the multivariate logistic regression analysis of the prognostic factors associated with 30-day mortality. AST intervention did not worsen the 30-day mortality after adjusting for the reported risk factors: age, malignancy, and inappropriate definitive therapy [25]. 


\section{Discussion}

Although the benefits of ASP have been reported, to our knowledge, this is the first report of the effect of ASP on anaerobic bacteremia. This study showed that AST could decrease the use of carbapenems without worsening patient outcomes. Moreover, although the use of TAZ/PIPC as an empiric therapy increased significantly after ASP implementation, the susceptibility rate of Bacteroides species to TAZ/PIPC did not change.

Antimicrobial resistance among anaerobic bacteria has been increasing similar to that among aerobic bacteria [12-14]. A surveillance study from Taiwan reported a significant increase in resistance to sulbactam/ampicillin and clindamycin among anaerobic bacteria [15]. Moreover, carbapenem-resistant anaerobic bacteria have been emerging $[15,16]$. The reported susceptibility rates of Bacteroides fragilis and the non-fragilis group to antimicrobials in Japan are sulbactam/ampicillin: $81.3 \%$ and 70.3\%, TAZ/PIPC: $90.6 \%$ and $100 \%$, cefmetazole: $75.0 \%$ and $29.7 \%$, flomoxef: $75.0 \%$ and $45.9 \%$, clindamycin: $56.3 \%$ and $35.1 \%$, and meropenem: $87.5 \%$ and $100 \%$, respectively [26]. Compared with this report, the susceptibility rates in our hospital were relatively conserved. Although the empiric use of TAZ/PIPC increased, the susceptibility rate of Bacteroides species to TAZ/PIPC did not deteriorate. 
This study showed a significant decrease in the definitive use of carbapenems.

Lately, there have been concerns about the rapid increase in carbapenem consumption

[1]. Carbapenem use is a known risk factor for carbapenem-resistant Enterobacteriaceae

(CRE) infections [27], and the importance of ASP to prevent the emergence of CRE is

evident [28]. Although there are concerns about the discontinuation and de-escalation of

carbapenems, it is reported that adequate discontinuation or de-escalation does not

worsen patient outcomes $[29,30]$. The results of this study support those of these

previous reports. A significant increase in the empiric use of TAZ/PIPC and a trend

toward an increase in the definitive use of TAZ/PIPC were also confirmed, probably

because TAZ/PIPC was used as an alternative to carbapenems for severe infections. The

duration of carbapenem and TAZ/PIPC therapy did not change significantly in the

post-intervention group. McLaughlin et al. reported that TAZ/PIPC use retains a negative relationship with the incidence of carbapenem-intermediate or -resistant Enterobacteriaceae [31]. Furthermore, $\mathrm{Ng}$ et al. reported that the empiric use of TAZ/PIPC is associated with fewer acquisitions of multidrug-resistant bacterial and fungal infections compared with the empiric use of carbapenems [32]. TAZ/PIPC is a reasonable choice as a carbapenem-sparing agent for severe anaerobic infections.

This study also demonstrated a significant increase in the de-escalation rate in 
the post-intervention group. Although sufficient evidence is lacking, de-escalation is regarded as an effective strategy to avoid both the failure of the initial therapy and the emergence of resistant bacteria. A systematic review conducted by Ohji et al. reported that de-escalation improved or did not change patient outcomes in various types of infections [24]. Regarding bacteremia, Shime et al. reported that de-escalation is associated with a trend toward lower mortality and treatment failure rates [33]. Our study showed that ASP could promote de-escalation and implied that de-escalation did not affect the outcomes of patients with anaerobic bacteremia or other infections.

A previous study showed that age, cancer history, and inappropriate definitive therapy are associated with mortality in anaerobic bacteremia [25]. Multivariate analysis found that these three factors and AST interventions are not associated with 30-day mortality. This demonstrates that our AST interventions did not worsen patient outcomes. The reason why inappropriate definitive therapy was not associated with 30-day mortality is obscure. A possible explanation is the improvement of surgical intervention and drainage techniques that remove the anaerobic environment. Further studies are warranted.

This study has several limitations. First, the sample size was limited because of the low incidence of anaerobic bacteremia. Second, this study was a single-center, 
retrospective, and observational study. Because the characteristics of the patients, resistance rates of bacteria, and isolation frequency might vary depending on the regions and hospitals, selection bias is inevitable. Therefore, multicenter trials are needed to eliminate these biases. Third, considering the relatively long term of observation, improvement in knowledge and surgical techniques may have influenced antibiotic selection and patient outcomes. Fourth, genetic identification of bacteria and susceptibility tests for bacteria other than Bacteroides species were not performed. Therefore, the identification of bacteria and the judgment of whether antimicrobial therapy was appropriate may be incorrect.

In conclusion, our ASP could decrease carbapenem consumption without worsening patient outcomes in anaerobic bacteremia. To further improve the clinical outcomes of anaerobic bacteremia, cooperation with attending physicians and prompt appropriate antimicrobial selection with reference to clinical backgrounds and microbiological examination are essential. 


\section{Acknowledgments}

We would like to thank Editage (www.editage.jp) for English language editing.

\section{Funding}

This work was financially supported by Grant-in-Aid for Scientific Research (C) from the Japan Society for the Promotion of Science [grant numbers 18K16185 and 19K08958] and the Research Program on Emerging and Reemerging Infectious Diseases of Japan Agency for Medical Research and Development (AMED) [grant numbers JP19fk0108094 and JP19fk0108052]. 


\section{References}

[1] E. Y. Klein, T.P. Van Boeckel, E.M. Martinez, S. Pant, S. Gandara, S.A. Levin, et al., Global increase and geographic convergence in antibiotic consumption between 2000 and 2015, Proc. Natl. Acad. Sci. U.S.A. 115 (2018) E3463-E3470. https://doi.org/10.1073/pnas.1717295115.

[2] E.C. Schuts, M.E.J.L. Hulscher, J.W. Mouton, C.M. Verduin, J.W.T.C. Stuart, H.W.P.M Overdiek, et al. Current evidence on hospital antimicrobial stewardship objectives: a systematic review and meta-analysis, Lancet Infect. Dis. 16 (2016) 847-856. https://doi.org/10.1016/S1473-3099(16)00065-7.

[3] I.M. Gould, Y. Lawes, Antibiotic stewardship: prescribing social norms, Lancet 387 (2016) 1699-1701. https://doi.org/10.1016/S0140-6736(16)00007-6.

[4] H. Grundmann, C. Glasner, B. Albiger, D.M. Aanensen, C.T. Tomlinson, A.T. Andrasević, et al., Occurrence of carbapenemase-producing Klebsiella pneumoniae and Escherichia coli in the European survey of carbapenemase-producing Enterobacteriaceae (EuSCAPE): a prospective, multinational study, Lancet Infect. Dis. 17 (2017) 153-163. https://doi.org/10.1016/S1473-3099(16)30257-2.

[5] Centers for Disease Control and Prevention. Antibiotic resistance threats in the United States, 2013. http://www.cdc.gov/drugresistance/threat-report-2013/ 
[6] C.M. Morel, E. Mossialos, Stoking the antibiotic pipeline, BMJ. 340 (2010) c2115. https://doi.org/10.1136/bmj.c2115.

[7] T.F. Barlam, S.E. Cosgrove, L.M. Abbo, C. MacDougall, A.N. Schuetz, E.J. Septimus, et al., Implementing an antibiotic stewardship program: guidelines by the Infectious Diseases Society of America and the Society for Healthcare Epidemiology of America, Clin. Infect. Dis. 62 (2016) e51-77. https://doi.org/10.1093/cid/ciw118.

[8] L. Blairon Y. De Gheldre, B. Delaere, A. Sonet, A. Bosly, Y. Glupczynski, A 62-month retrospective epidemiological survey of anaerobic bacteremia in a universal hospital, Clin. Microbiol. Infect. 12 (2006) 527-532. https://doi.org/10.1111/j.1469-0691.2006.01407.x

[9] D.P. Lombardi, N.C. Engleberg, Anaerobic bacteremia: incidence, patient characteristics, and clinical significance, Am. J. Med. 92 (1992) 53-60. https://doi.org/10.1016/0002-9343(92)90015-4.

[9] D.P. Lombardi, N.C. Engleberg, Anaerobic bacteremia: incidence, patient characteristics, and clinical significance, Am. J. Med. 92 (1992) 53-60. https://doi.org/10.1016/0002-9343(92)90015-4.

[11] B. Lassmann, D.R. Gustafson, C.M. Wood, J.E. Rosenblatt, Reemergence of 
anaerobic bacteremia, Clin. Infect. Dis. 44 (2007) 895-900.

https://doi.org/10.1086/512197.

[12] D.W. Hecht, Prevalence of antibiotic resistance in anaerobic bacteria: worrisome developments, Clin. Infect. Dis. 39 (2004) 92-97. https://doi.org/10.1086/421558.

[13] W. Jamal, M. Shahin, V.O. Rotimi, Surveillance and trends of antimicrobial resistance among clinical isolates of anaerobes in Kuwait hospitals from 2002 to 2007. Anaerobe 16 (2010) 1-5. https://doi.org/10.1016/j.anaerobe.2009.04.004.

[14] J.A. Karlowsky, A.J. Walkty, H.J. Adam, M.R. Baxter, D.J. Hoban, G.G. Zhanel, Prevalence of antimicrobial resistance among clinical isolates of Bacteroides fragilis group in Canada in 2010-2011: CANWARD surveillance study. Antimicrob. Agents Chemother. 56 (2012) 1247-1252. https://doi.org/10.1128/AAC.05823-11.

[15] C.Y. Liu, Y.T. Huang, C.H. Liao, L.C. Yen, H.Y. Lin, P.R. Hsueh, Increasing trends in antimicrobial resistance among clinically important anaerobes and Bacteroides fragilis isolates causing nosocomial infections: emerging resistance to carbapenems, Antimicrob. Agents Chemother. 52 (2008) 3161-3168. https://doi.org/10.1128/AAC.00355-08.

[16] Y. Shafquat, K. Jabeen, J. Farooqi, K. Mehmood, S. Irfan, R. Hasan, et al., Antimicrobial susceptibility against metronidazole and carbapenem in clinical 
anaerobic isolates from Pakistan, Antimicrob. Resist. Infect. Control 8 (2019) 99. https://doi.org/10.1186/s13756-019-0549-8.

[17] S. Karanika, S. Paudel, C. Grigoras, A. Kalbasi, E. Mylonakis, Systematic review and meta-analysis of clinical and economic outcomes from the implementation of hospital-based antimicrobial stewardship programs, Antimicrob. Agents Chemother. 60 (2016) 4840-4852. https://doi.org/10.1128/AAC.00825-16.

[18] D. Baur, B.P. Gladstone, F. Burkert, E. Carrara, F. Foschi, S. Döbele, et al., Effect of antibiotic stewardship on the incident of infection and colonization with antibiotic-resistant bacteria and Clostridium difficile infection: a systematic review and meta-analysis, Lancet Infect. Dis. 17 (2017) 990-1001. https://doi.org/10.1016/S1473-3099(17)30325-0.

[19] K. Yamada, W. Imoto, K. Yamairi, W. Shibata, H. Namikawa, N. Yoshii, et al., The intervention by an antimicrobial stewardship team can improve clinical and microbiological outcomes of resistant gram-negative bacteria, J. Infect. Chemother. 25 (2019) 1001-1006. https://doi.org/10.1016/j.jiac.2019.05.026.

[20] T. Umemura, Y. Hamada, Y. Yamagishi, H. Suematsu, H. Mikamo, Clinical characteristics associated with mortality of patients with anaerobic bacteremia, Anaerobe 39 (2016) 45-50. https://doi.org/10.1016/j.anaerobe.2016.02.007. 
[21] S.E. Sharp, J.C. McLaughlin, J.M. Goodman, J. Moore, S.M. Spanos, D.W. Keller 3rd,et al., Clinical assessment of anaerobic isolates from blood cultures, Diagn. Microbiol. Infect. Dis. 17 (1993) 19-22. https://doi.org/10.1016/0732-8893(93)90064-e.

[22] T. Kimura, A. Uda, T. Sakaue, K. Yamashita, T. Nishioka, S. Nishimura, et al., Long-term efficacy of comprehensive multidisciplinary antibiotic stewardship programs centered on weekly prospective audit and feedback, Infection 46 (2018) 215-224. https://doi.org/10.1007/s15010-017-1099-8.

[23] N.Y. Lee, C.C. Lee, W.H. Huang, K.C. Tsui, P.R. Hsueh, W.C. Ko, Cefepime therapy for monomicrobial bacteremia caused by cefepime-susceptible extended-spectrum beta-lactamase-producing Enterobacteriaceae: MIC matters, Clin. Infect. Dis. 56 (2013) 488-495. https://doi.org/10.1093/cid/cis916.

[24] G. Ohji, A. Doi, S. Yamamoto, K. Iwata, Is de-escalation of antimicrobials effective? A systematic review and meta-analysis, Int. J. Infect. Dis. 49 (2016) 7179. https://doi.org/10.1016/j.ijid.2016.06.002.

[25] R. Robert, A. Deraignac, G. Le Moal, S. Ragot, G. Grollier, Prognostic factors and impact of antibiotherapy in 117 cases of anaerobic bacteraemia, Eur. J. Clin. 
Microbiol. Infect. Dis. 27 (2008) 671-678.

https://doi.org/10.1007/s10096-008-0487-5.

[26] Y. Takesue, S. Kusachi, H. Mikamo, J. Sato, A. Watanabe, H. Kiyota, et al., Antimicrobial susceptibility of common pathogens isolated from postoperative intra-abdominal infections in Japan, J. Infect. Chemother. 24 (2018) 330-340. https://doi.org/10.1016/j.jiac.2018.02.011.

[27] Q. Wang, Y. Zhang, X. Yao, H. Xian, Y. Liu, H. Li, et al., Risk factors and clinical outcomes for carbapenem-resistant Enterobacteriaceae nosocomial infections, Eur. J. Clin. Microbiol. Infect. Dis. 35 (2016) 1679-1689. https://doi.org/10.1007/s10096-016-2710-0.

[28] D. Marchaim, T. Chopra, A. Bhargava, C. Bogan, S. Dhar, K. Hayakawa, et al., Recent exposure to antimicrobials and carbapenem-resistant Enterobacteriaceae: the role of antimicrobial stewardship, Infect. Control. Hosp. Epidemiol. 33 (2012) 817-830. https://doi.org/10.1086/666642.

[29] CB Teng, TM Ng, MW Tan, SH Tan, M Tay, SF Lim, et al., Safety and effectiveness of improving carbapenem use via prospective review and feedback in a multidisciplinary antimicrobial stewardship programme, Ann. Acad. Med. Singapore 44 (2015) 19-25. 
[31] M. McLaughlin, M.R. Advincula, M. Malczynski, C. Qi, M. Bolon, M.H. Scheetz, Correlations of antibiotic use and carbapenem resistance in Enterobacteriaceae, Antimicrob. Agents Chemother. 57 (2013) 5131-5133. https://doi.org/10.1128/AAC.00607-13.

[32] T.M. Ng, W.X. Khong, P.N. Harris, P.P. De, A. Chow, P.A. Tambyah, et al., Empiric piperacillin-tazobactam versus carbapenems in the treatment of bacteraemia due to extended-spectrum beta-lactamase-producing Enterobacteriaceae, PLoS One 11 (2016) e0153696. https://doi.org/10.1371/journal.pone.0153696.

[33] N. Shime, S. Satake, N. Fujita, De-escalation of antimicrobials in the treatment of bacteraemia due to antibiotic-sensitive pathogens in immunocompetent patients, Infection 39 (2011) 319-325. https://doi.org/10.1007/s15010-011-0116-6. 
Table 1. Characteristics of patients with anaerobic bacteremia

\begin{tabular}{|c|c|c|c|}
\hline & $\begin{array}{l}\text { Pre-intervention } \\
(n=53)\end{array}$ & $\begin{array}{l}\text { Post-intervention } \\
(n=67)\end{array}$ & P-value \\
\hline Age $($ mean $\pm S D)$, years & $64.21 \pm 17.89$ & $63.10 \pm 20.03$ & 0.751 \\
\hline Age $\geq 65$ & $26(49.0 \%)$ & $38(56.7 \%)$ & 0.515 \\
\hline Male & $30(56.6 \%)$ & $40(85.1 \%)$ & 0.877 \\
\hline $\begin{array}{l}\text { White blood cell count } \\
\text { (mean } \pm \text { SD) }\end{array}$ & $11773 \pm 1072$ & $12013 \pm 11.82$ & 0.909 \\
\hline C-reactive protein (mean \pm & $12.53 \pm 10.06$ & $13.00 \pm 9.55$ & 0.794 \\
\hline $\mathrm{SD})$ & & & \\
\hline SOFA score $($ mean \pm SD) & $3.85 \pm 4.28$ & $3.07 \pm 3.42$ & 0.273 \\
\hline Abdominal surgery & $33(62.2 \%)$ & $43(64.2 \%)$ & 0.121 \\
\hline Malignancy & $33(62.2 \%)$ & $43(64.2 \%)$ & 0.980 \\
\hline Hematological & $7(13.2 \%)$ & $5(7.5 \%)$ & 0.462 \\
\hline Solid & $25(47.2 \%)$ & $38(56.7 \%)$ & 0.392 \\
\hline Diabetes mellitus & $11(20.8 \%)$ & $13(19.4 \%)$ & 1.000 \\
\hline Chemotherapy & $11(20.8 \%)$ & $10(14.9 \%)$ & 0.553 \\
\hline Chronic kidney disease & $6(11.3 \%)$ & $11(16.4 \%)$ & 0.595 \\
\hline Use of immunosuppressants & $9(17.0 \%)$ & $3(4.5 \%)$ & 0.050 \\
\hline
\end{tabular}




\begin{tabular}{|c|c|c|c|}
\hline ICU admission & $7(13.2 \%)$ & $18(26.9 \%)$ & 0.109 \\
\hline Nosocomial & $34(64.2 \%)$ & $39(58.2 \%)$ & 0.636 \\
\hline Multibacterial bacteremia & $9(17.0 \%)$ & $23(34.3 \%)$ & 0.054 \\
\hline \multicolumn{4}{|l|}{ Source of bacteremia } \\
\hline Intra-abdominal cavity & $14(26.4 \%)$ & $21(31.3 \%)$ & 0.698 \\
\hline Hepatobiliary tract & $4(7.5 \%)$ & $10(14.9 \%)$ & 0.335 \\
\hline Gastrointestinal tract & $3(5.7 \%)$ & $2(3.0 \%)$ & 0.788 \\
\hline Skin & $4(7.5 \%)$ & $6(9.0 \%)$ & 1.000 \\
\hline Urinary tract & $0(0.0 \%)$ & $3(4.5 \%)$ & 0.331 \\
\hline Respiratory tract & $2(3.8 \%)$ & $1(1.5 \%)$ & 0.837 \\
\hline Others & $3(5.7 \%)$ & $6(9.0 \%)$ & - \\
\hline Unknown & $23(43.3 \%)$ & $18(26.9 \%)$ & - \\
\hline \multicolumn{4}{|l|}{ Inappropriate empiric } \\
\hline & $10(18.9 \%)$ & $11(16.4 \%)$ & 0.913 \\
\hline \multicolumn{4}{|l|}{ therapy } \\
\hline \multicolumn{4}{|l|}{ Inappropriate definitive } \\
\hline & $8(15.1 \%)$ & $6(9.0 \%)$ & 0.416 \\
\hline \multicolumn{4}{|l|}{ therapy } \\
\hline 30-day mortality & $7(13.2 \%)$ & $7(10.4 \%)$ & 0.873 \\
\hline
\end{tabular}

SD: standard deviation, SOFA: Sequential Organ Failure Assessment, ICU: intensive care unit 
Table 2. Anaerobic bacteria detected in the blood cultures

\begin{tabular}{|c|c|c|c|}
\hline Bacteria & Pre-intervention $(\mathrm{n}=53)$ & Post-intervention(n=67) & P-value \\
\hline Bacteroides fragilis & 18 & 11 & \\
\hline \multicolumn{4}{|l|}{ Bacteroide } \\
\hline & 7 & 3 & \\
\hline \multicolumn{4}{|l|}{ thetaiotaomicron } \\
\hline Bacteroide uniformis & 2 & 4 & \\
\hline Bacteroide vulgatus & 0 & 1 & \\
\hline Bacteroide stercolis & 1 & 0 & \\
\hline Bacteroide denticola & 1 & 0 & \\
\hline Bacteroide capillosus & 1 & 0 & \\
\hline Bacteroides species & 1 & 4 & \\
\hline Total & $31(58.5 \%)$ & $23(34.3 \%)$ & 0.014 \\
\hline Clostridium perfringens & 3 & 4 & \\
\hline Clostridium butyricum & 3 & 3 & \\
\hline Clostridium tertium & 1 & 3 & \\
\hline Clostridium ramosum & 1 & 1 & \\
\hline Clostridium paraputrificum & 0 & 2 & \\
\hline Clostridium bifermentans & 0 & 1 & \\
\hline
\end{tabular}




\begin{tabular}{|c|c|c|c|}
\hline Clostridium glycolicum & 0 & 1 & \\
\hline Clostridium species & 4 & 2 & \\
\hline Total & $12(22.6 \%)$ & $17(25.4 \%)$ & 0.895 \\
\hline Fusobacterium nucleatum & 4 & 8 & \\
\hline Fusobacterium varium & 1 & 1 & \\
\hline \multicolumn{4}{|l|}{ Fusobacterium } \\
\hline & 0 & 1 & \\
\hline \multicolumn{4}{|l|}{ necrophorum } \\
\hline Fusobacterium mortiferum & 0 & 1 & \\
\hline Fusobacterium species & 0 & 1 & \\
\hline Total & $5(9.4 \%)$ & $12(17.9 \%)$ & 0.292 \\
\hline Peptostreptococcus micros & 1 & 2 & \\
\hline \multicolumn{4}{|l|}{ Peptostreptococcus } \\
\hline & 1 & 1 & \\
\hline \multicolumn{4}{|l|}{ anaerobius } \\
\hline \multicolumn{4}{|l|}{ Peptostreptococcus } \\
\hline & 0 & 1 & \\
\hline \multicolumn{4}{|l|}{ productus } \\
\hline \multicolumn{4}{|l|}{ Peptostoreptococcus } \\
\hline & 6 & 3 & \\
\hline \multicolumn{4}{|l|}{ species } \\
\hline Total & $8(15.1 \%)$ & $7(10.4 \%)$ & 0.627 \\
\hline
\end{tabular}




\begin{tabular}{llll} 
Prevotella oralis & 0 & 2 & \\
Prevotella Buccae & 0 & 1 & \\
Prevotella species & 0 & 3 & 0.290 \\
Total & $0(0 \%)$ & $6(9.0 \%)$ & 0.382 \\
\hline Veillonella species & $1(1.9 \%)$ & $4(6.0 \%)$ & 0.254 \\
\hline Propionibacterium species & $0(0 \%)$ & $3(4.5 \%)$ & 1.000 \\
\hline Porphyromonas & $0(0 \%)$ & $1(1.5 \%)$ & \\
\hline endodontalis & & & \\
\hline
\end{tabular}


Table 3. Empiric and definitive therapy

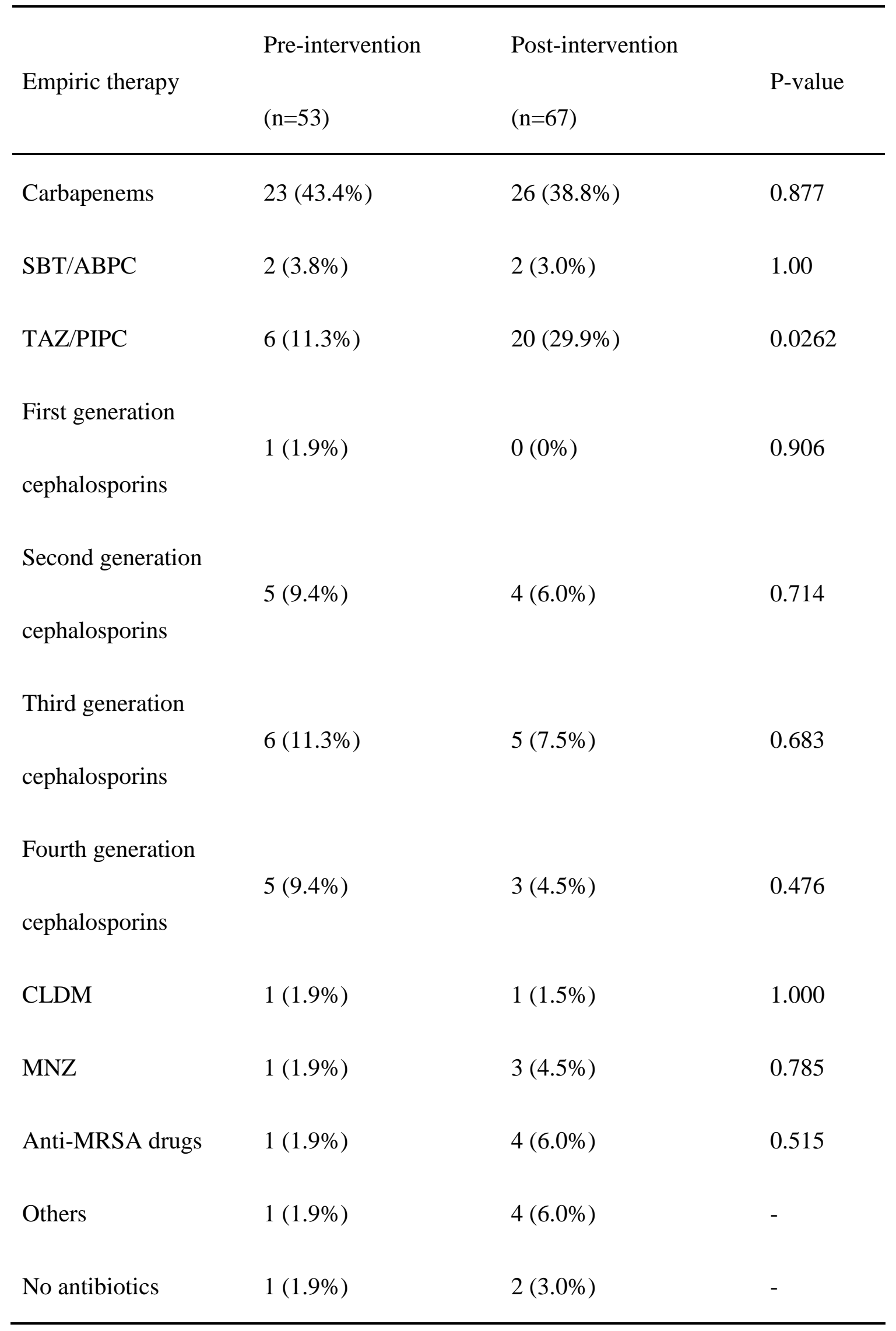




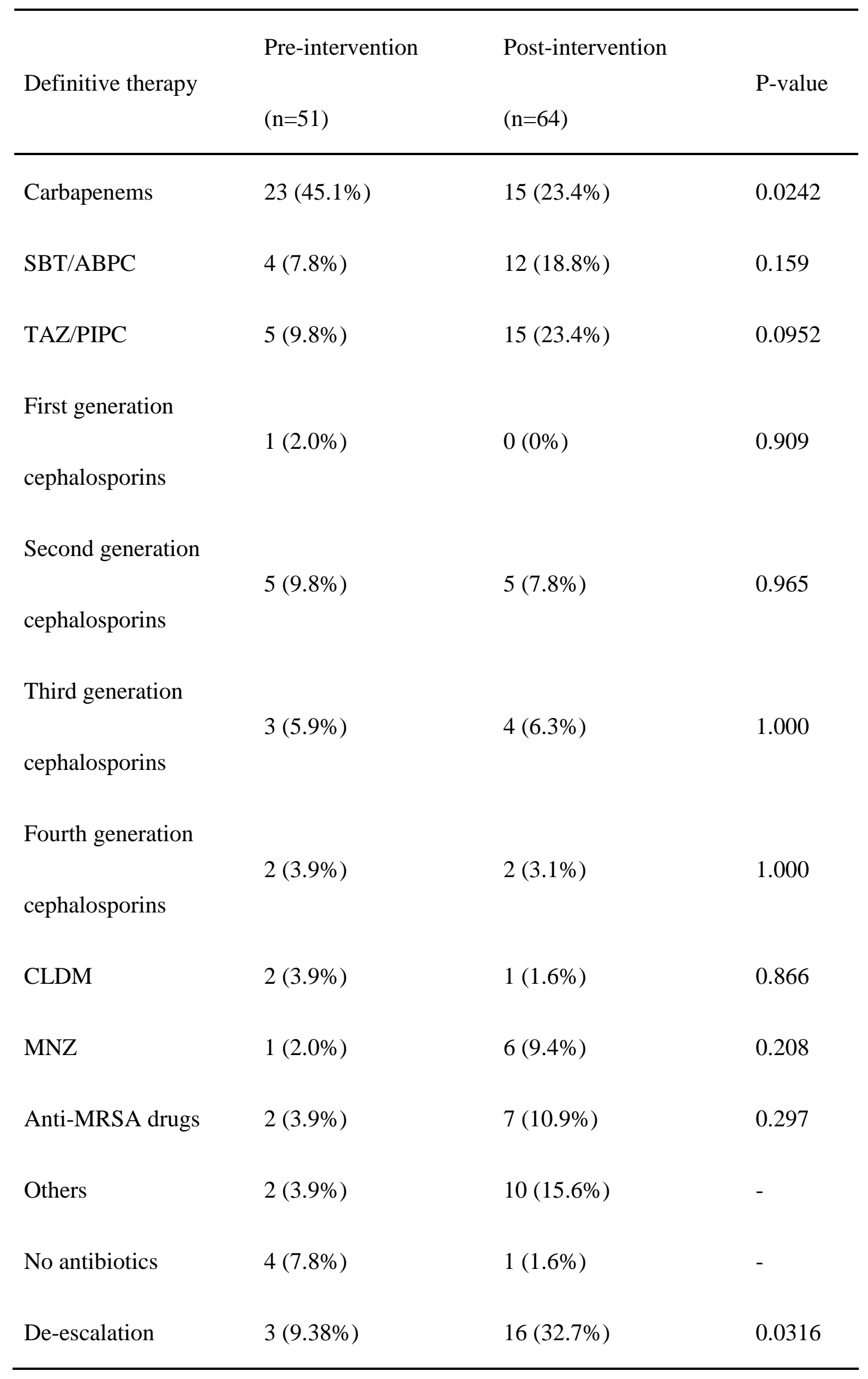


Two and three patients dropped out before definitive therapy in the pre-intervention group and the post-intervention group, respectively.

SBT/ABPC: sulbactam/ampicillin, TAZ/PIPC: tazobactam/piperacillin, CLDM:

clindamycin, MNZ: metronidazole, MRSA: methicillin-resistant Staphylococcus aureus 
Table 4. Duration of carbapenem and TAZ/PIPC therapy

\begin{tabular}{llll}
\hline & Pre-intervention & Post-intervention & P-value \\
\hline Carbapenem (mean \pm SD) & $11.8 \pm 9.35$ & $8.42 \pm 4.32$ & 0.0733 \\
TAZ/PIPC (mean \pm SD) & $6.33 \pm 4.03$ & $7.42 \pm 4.37$ & 0.587 \\
\hline
\end{tabular}

TAZ/PIPC: tazobactam /piperacillin, SD: standard deviation

Table 5. Prognostic factors associated with 30-day mortality in multivariate logistic regression analysis

$$
\text { Odds ratio }(95 \% \mathrm{CI}) \quad \mathrm{P} \text {-value }
$$

\begin{tabular}{llc}
\hline Intervention & $0.536(0.147-1.95)$ & 0.343 \\
Age $\geq 65$ years & $3.35(0.806-13.9)$ & 0.0962 \\
Malignancy & $1.55(0.367-6.58)$ & 0.550 \\
Inappropriate definitive therapy & $\mathrm{ND}$ & $\mathrm{ND}$ \\
\hline
\end{tabular}

Three patients dropped out within 30 days after bacteremia onset and could not be tracked.

CI: confidence interval, ND: not detected 


\section{Figure legend}

Fig 1. Susceptibility of (A) Bacteroides species, (B) Bacteroides fragilis, and (C) the non-fragilis group.

SBT/ABPC: sulbactam/ampicillin, TAZ/PIPC: tazobactam/piperacillin, CMZ:

cefmetazole, FMOX: flomoxef, CLDM: clindamycin, MEPM: meropenem, IPM:

imipenem 
(A)

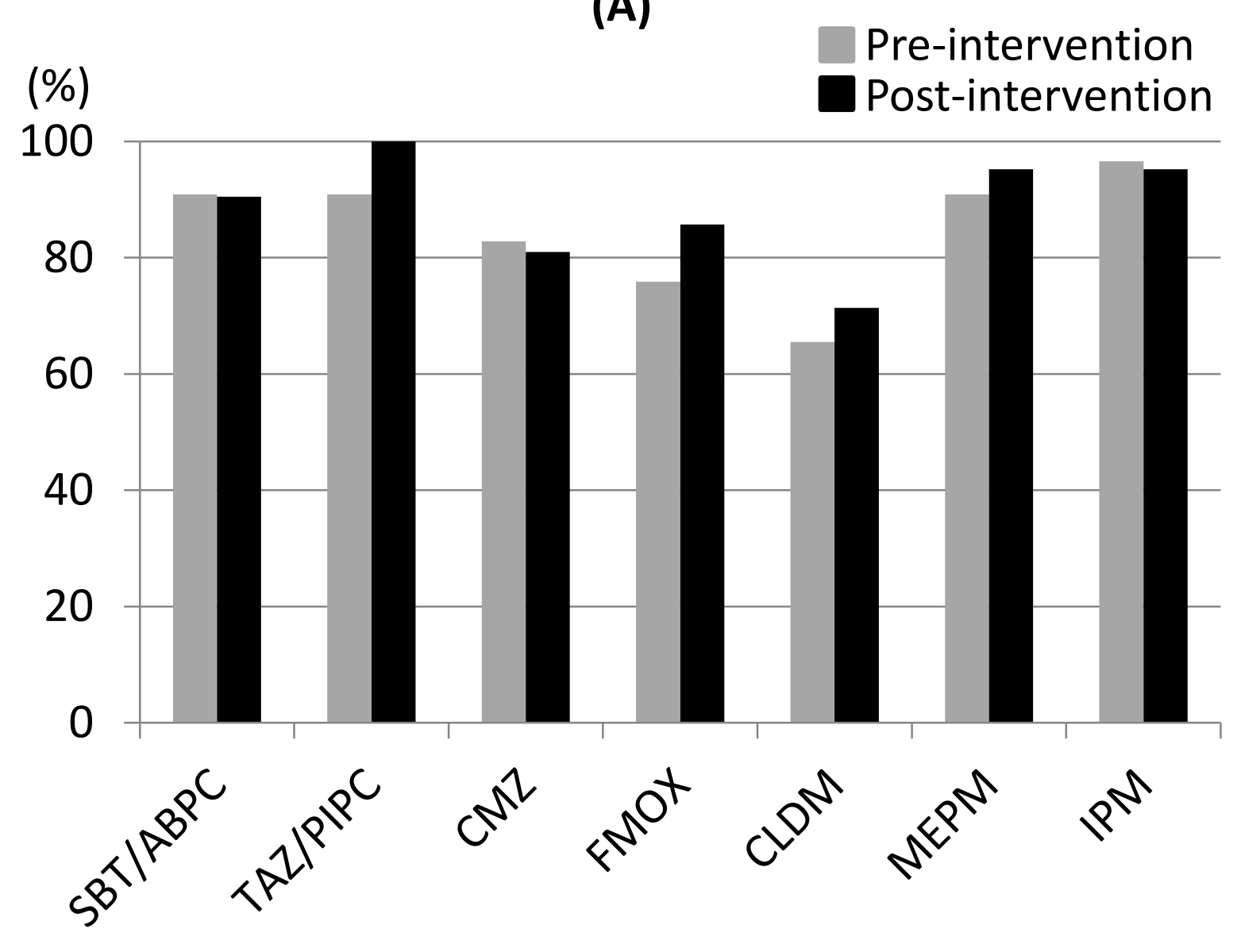

Figure 1 
(B)

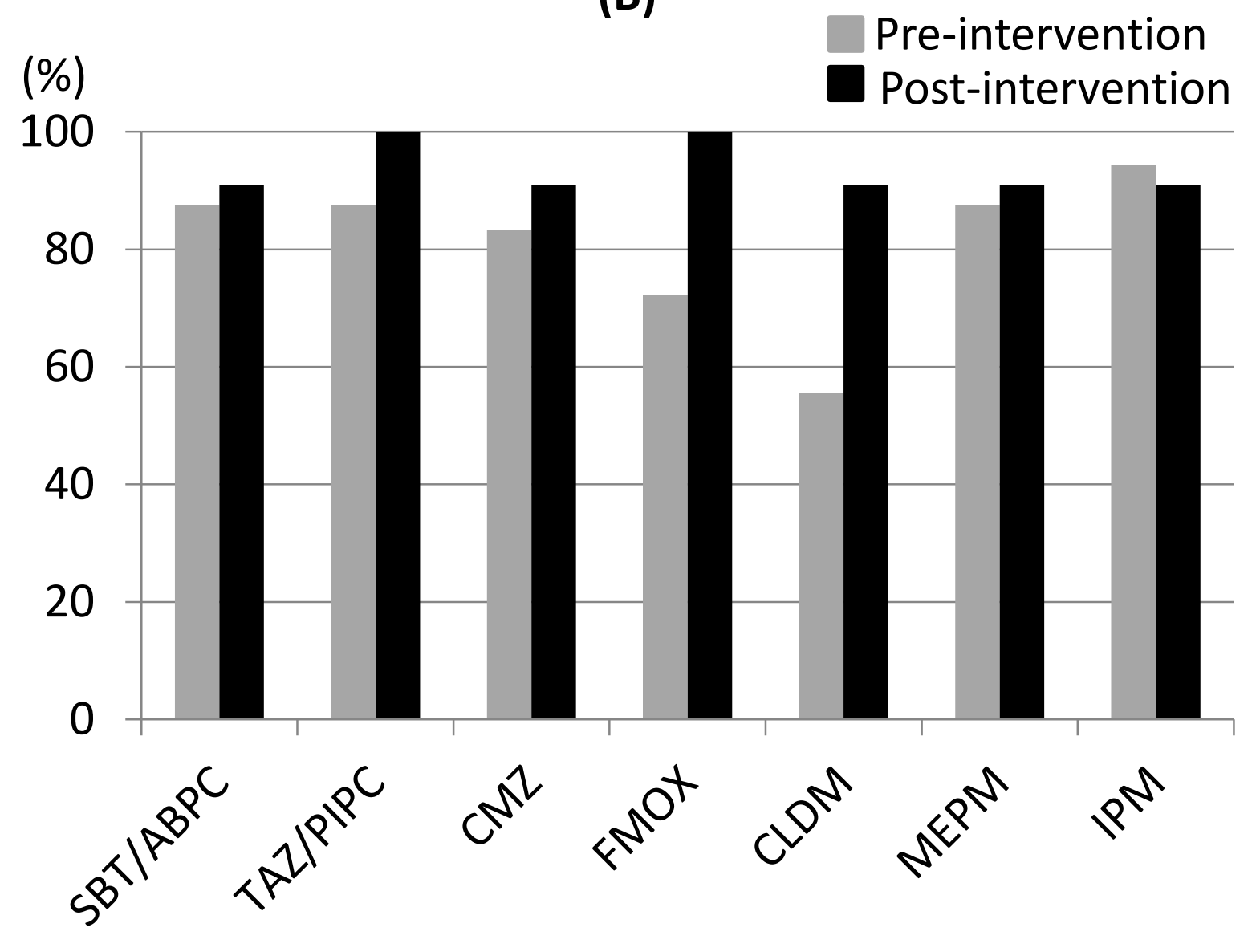

Figure 1 
(C)

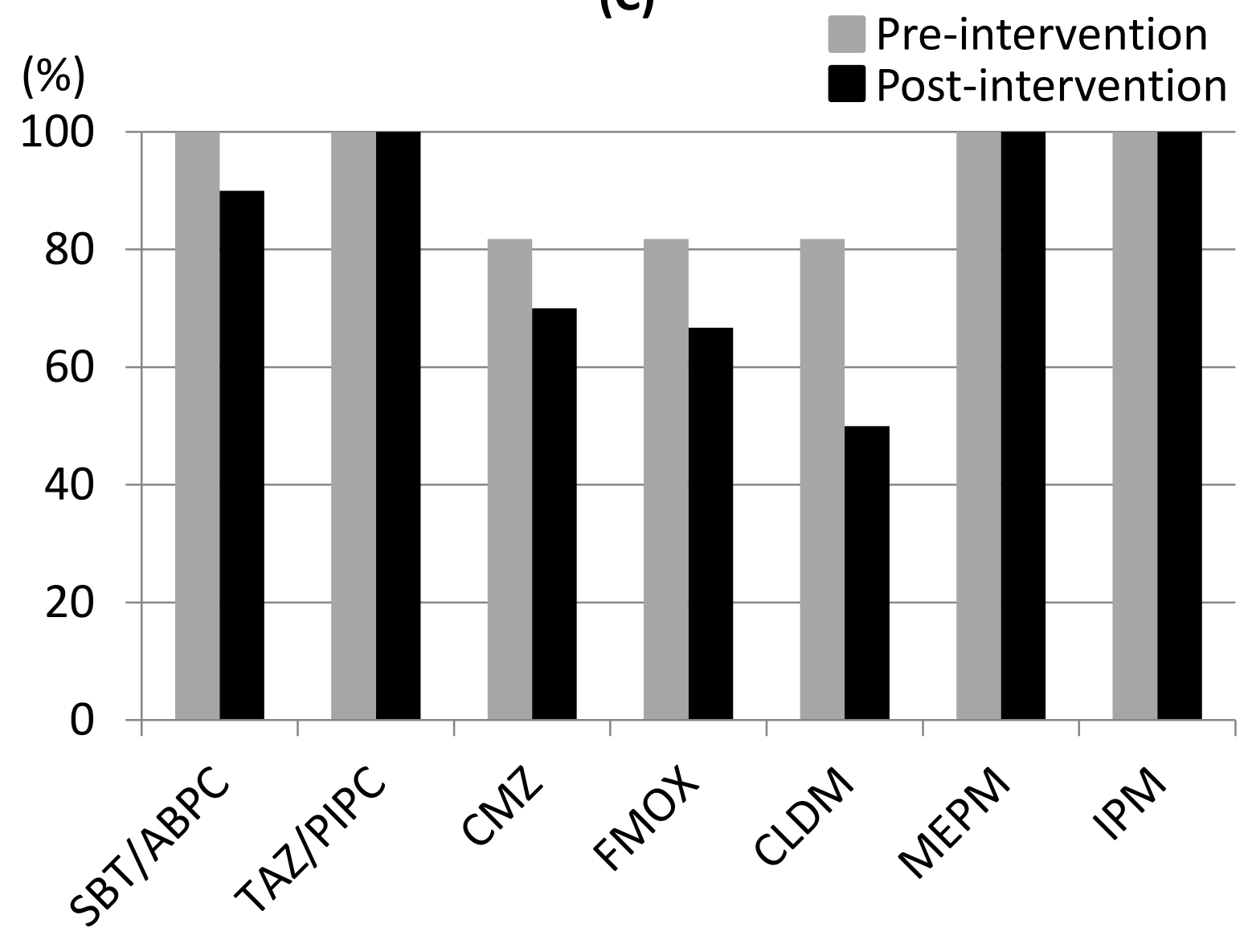

Figure 1 